\title{
Audioguides. Oder „Mit Ohren sehen“! \\ Zur Übersetzung der Textsorte „Audioguide“ als Texte der Kunstkommunikation am Beispiel des Textes „Früher Lagerhalle, heute Moschee“
}

\author{
Dr. Mohamed Nasef
}

\begin{abstract}
Abstrakt:
Die deutsche Sprache verfügt über zwanzig Textsorten. Der Audioguide als Textsorte, welcher im Mittelpunkt dieser Studie liegt, ist relativ neu und kann die Rolle eines menschlichen Museumsführers ersetzen. Nach dem Motto „Mit den Ohren sehen" werden Audioguides im musealen Umfeld bzw. in Kulturprojekten eingesetzt. Dabei handelt es sich um akustisch eingerichtete Hörgeräte für die Verwendung bei Ausstellungsbesucher-Rundgängen. Der Textinhalt der Audioguides ist so eingerichtet, dass die Informationen bildhaft und nutzergerecht vermittelt werden. Diese Studie diskutiert die Regeln, die zu befolgen sind, um den Audioguide-Text dem Besucher genaue Details über Exponate zu vermitteln.

Dabei thematisiert diese Untersuchung anhand eines Audioguide-Textes „Früher Lagerhalle, heute Moschee“ folgende Punkte: 1. Die schriftliche Wiedergabe des gehörten Textes und seine Übersetzung ins Arabische. 2. Diskussion über die arabische Übersetzung. 3. Analyse des Audioguides nach den Kriterien der sprachlichen Merkmale.
\end{abstract}

Schlüsselwörter: Audioguide - Übersetzung - Museum Kunstkommunikation. 


\section{مجلة وادي النيل للاراسات والبحوث الإنسانية والاجتماعية والتربوية (مجلة علمية محكمة)}

(ISSN : 2536 - 9555)

\section{المرثد الصوتى أو الرؤية عن طريق الاستماع}

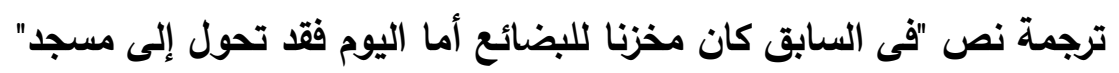

كنص لفن التواصل المسموع

د. محمد محمود ناصف

مدرس بقسم اللغة الألمانية كلية اللغات والترجمة جامعة ج اكتوبر

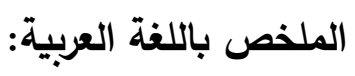

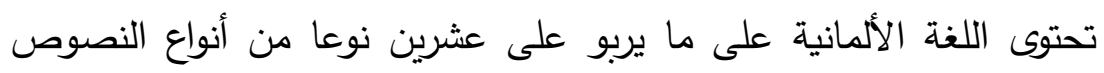

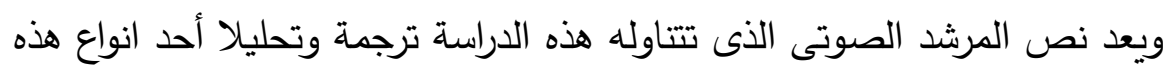

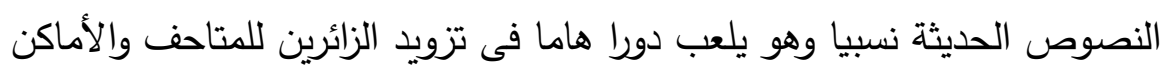

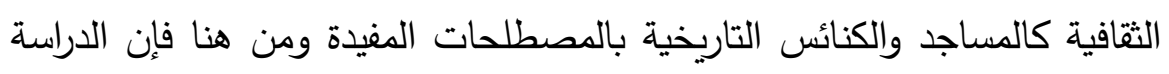

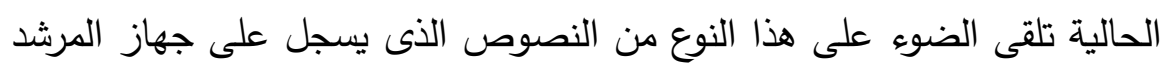

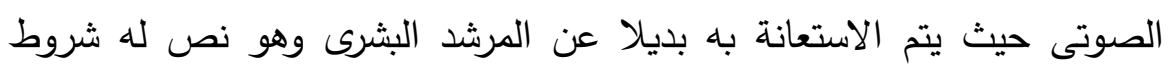

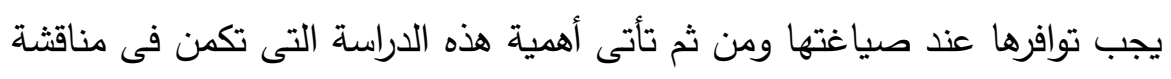

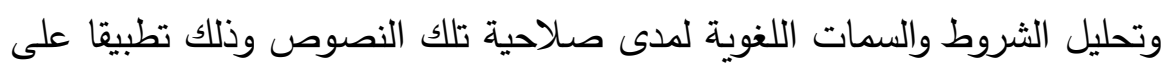

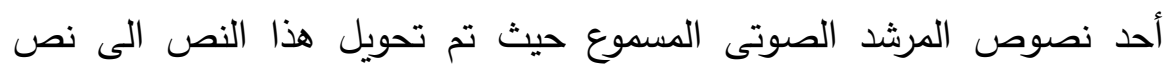

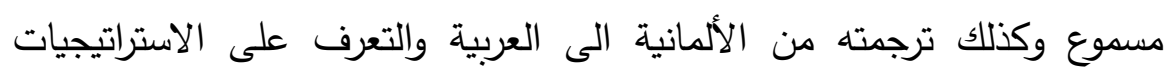

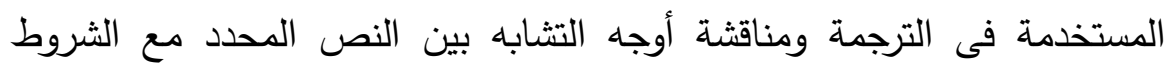

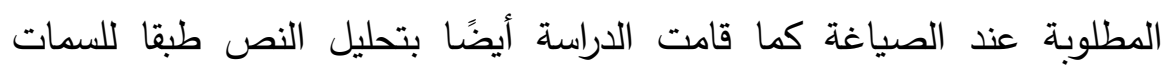
اللغوية.

الكلمات المفتاحية: المرشد الصوتى - الترجمة - المتاحف - فن التواصل 
Audioguides. Oder „Mit Ohren sehen“!

Zur Übersetzung der Textsorte „Audioguide“ als Texte der Kunstkommunikation am Beispiel des Textes „Früher Lagerhalle, heute Moschee“6

Dr. Mohamed Nasef

مجلة وادي النيل للاراسات والبحوث الإنسانية والاجتماعية والتربوية (مجلة علمية محكمة)

Audioguides. Oder „Mit Ohren sehen“"

Zur Übersetzung der Textsorte „Audioguide“ als Texte

der Kunstkommunikation am Beispiel des Textes „Früher

Lagerhalle, heute Moschee“

Dr. Mohamed Nasef

\section{Einleitung}

Der vorliegende Beitrag beschäftigt sich mit der Übersetzung der Textsorte „Audioguide“ bzw. „Audioführer". Diese kontextgebundene Textsorte ist relativ neu und kann die Rolle eines menschlichen Museumsführers ersetzen. Dabei geht es um Texte, die meist von einem Experten ${ }^{1}$ im Voraus formuliert und dann von einem Sprecher/ einer Sprecherin mündlich verwirklicht werden. Meistens sind Audioguides informative, kommunikative Texte, die gemäß dem Konzept der „echten Simultankommunikation“ von Fandyrich/Thurmair (2016: 381) nicht allein rezipiert, sondern während einer Stadtführung oder in einer Museumausstellung eingesetzt werden. Weiterhin erfüllen sie didaktische Funktionen, wie bspw. Beschreiben, Deuten, Erläutern und Bewerten (vgl. Fandrych/Thumair 2011:73). Poppe (2013) zufolge „liefert [der Audioguide] ergänzende Informationen zu einer Ausstellung, erläutert einzelne Exponate und vermittelt Hintergrundwissen dazu“ (Poppe 2013: 40). Laut Dech (2004:11) zielen Audioguides auf „eine lehrreiche und dialogische Interaktion zwischen dem Besucher und ausgewählten Ausstellungsstücken“ ab: Sie werden konzipiert, um dem Besucher

${ }^{1}$ Aus Gründen der Lesbarkeit wird nur die männliche Form verwendet und Frauen sind mitgemeint. 
(ISSN : 2536 - 9555)

den Inhalt der Ausstellungsstücke verständlich zu machen. Da Sprache und Kultur sich gegenseitig beeinflussen, reflektieren Audioguides kulturgeprägte Spezifika der Sprache. Vor diesem Hintergrund sind Audioguides speziell auf folgende sprachliche Ausdrucksmittel angewiesen: Attribute, Tempusgebrauch, didaktische Elemente zur räumlichen Orientierung, Referenzmittel des direkten Adressatenbezugs, rhetorische Fragen und Negationen (vgl. ebd. 84ff.).

\section{Ausgangssituation und Zielsetzung}

Der vorliegende Beitrag setzt sich zum Ziel, die sprachlichen Sachinformationen von Audioguides und ihrer Übersetzung vor dem Hintergrund zentraler Erkenntnisse aus der Übersetzungsforschung zu diskutieren. In einem ersten Schritt wird die Definition des Begriffs „Audioguide“ geklärt. Der Begriff "Audioguide“ ist ein zusammengesetztes Wort, das aus dem lateinischen Wort „Audio“: (Ich höre) und aus dem englischen Wort „Guide“: (Führer) besteht. Mit diesem Begriff werden akustische Führungen, vor allem durch künstliche Museen und Ausstellungen, bezeichnet (vgl. Fandrych/Thumair 2011: 73). Audioguides sind Tonaufnahmen, die auf entsprechenden Geräten oder Mobiletelefonen abgespielt werden können. Audioguides können jedoch ebenfalls in anderen Kontexten, entweder als begleitende oder als alleinige Informationsquellen für Stadtführungen verwendet werden (vgl. ebd.: 41).

Audioguides können auch in Form von Podcasts ${ }^{2}$ veröffentlicht werden. Weiterhin ist es möglich, dass Audioguides nicht von

2. Der Begriff „Podcast“ ist ein Kunstwort, das sich aus dem englischen Wort „Pod“, abgeleitet vom sogenannten i-Pod des Unternehmens Apple, einem Gerät zum Abspielen von MP3-Dateien, und dem ebenfalls englischen Wort „cast“, einer Abkürzung des Begriffs Broadcast (dt. Rundfunk), zusammensetzt. Der Begriff selbst ,steht für die Verbreitung digitaler (Ton-) Aufzeichnungen über das Internet für die Nutzung mit tragbaren Abspielgeräten“" (Meier 2007: 91) 
Audioguides. Oder „Mit Ohren sehen“!

Zur Übersetzung der Textsorte „Audioguide“ als Texte der Kunstkommunikation am Beispiel des Textes „Früher Lagerhalle, heute Moschee“6

Dr. Mohamed Nasef

مجلة وادي النيل للاراسات والبحوث الإنسانية والاجتماعية والتربوية (مجلة علمية محكمة)

professionellen Anbietern bereitgestellt werden, sondern von Amateuranbietern, etwa von einer Schulklasse oder von einer Gruppe Hochschulstudenten. Diese Audioguides werden als „nutzergenerierte Audioguides“ bezeichnet. Sie haben sowohl im schulischen als auch im hochschulischen Bereich einen großen didaktischen Stellenwert

Als allgemeine Zielgruppe für Audioguides gelten ,alle Besucher". In Betracht gezogen werden dabei vielfältige Besuchergruppen wie Familien mit Kindern, Jugendliche (weiblich und männlich), Fachleute mit und ohne Vorwissen (Fachpublikum) und Laienpublikum, sowie Menschen mit Lernschwierigkeiten oder geistiger Behinderung. Die Entscheidung über die Zielgruppe beeinflusst die Gestaltung, den Stil sowie die Informationstiefe der Audioguides (vgl. Vorwerk/Medien 2001: 7). Bezüglich ihrer Struktur kommen Audioguides in zwei Formen vor: lineare und punktuelle Form. Bei einem linearen Typ ist die Reihenfolge der gehörten Texte im Vorhinein festgelegt. Die Inhalte bauen daher aufeinander auf. Im Gegensatz dazu haben Besucher bei punktuellen Audioguides die freie Wahl: Sie können die Texte nach eigenem Interesse zu hören, indem sie den entsprechenden Track anklicken. Bei dieser Variante stehen die Texte nicht miteinander in Beziehung. Stattdessen soll jeder Text an sich, ohne direkten Bezug zu einem anderen Text, verständlich sein. Die meisten Besucher favorisieren diese Variante, da sie die Inhalte nach ihrer Wahl hören können (vgl. ebd.: 8).

In den letzten Ausführungen haben wir einen Überblick über Audioguides als Untersuchungsgegenstand erhalten. Im Folgenden werden die Kommunikationssituation, die Textstruktur und ihre kennzeichnenden Merkmale unter die Lupe genommen. 
مجلة وادي النيل للار اسات والبحوث الإنسانية والاجتماعية والتربوية (مجلة علمية محكمة)

(ISSN : 2536 - 9555)

\section{Forschungsstand}

Mit dem wissenschaftlichen Feld „Audioguide“ hat sich eine nicht geringe Anzahl sowohl theoretischer als auch empirischer Studien beschäftigt. In seinem Beitrag "Aufmerksames Sehen" hat Dech (2004) ein experimentelles Konzept zur Gestaltung von Audioguides erstellt, mit dessen Hilfe Ausstellungsbesucher zur selbständigen Beobachtung und Diskussion mit Kunstwerken angeregt werden und die zu direkten direkte Fragen motivieren können. Der Sprachwissenschaftler Hausendorf (2005/2011/2016) hat sich mit dem Medium Audioguide einerseits, sowie der Multimodalität und Wissenskommunikation in Kunstausstellungen andererseits befasst. In seinem Beitrag „Kunstkommunikation“ unterscheidet er fünf „kommunikative Aufgaben“, die für ihn das Grundgerüst des Sprechens über Kunst ausmachen (vgl. Hausendorf 2011: 518). Das Hauptanliegen dieses Beitrags beschränkt sich jedoch auf die Betrachtung des Phänomens „Audioguide“ aus einer analytischen Perspektive.

Einen Überblick über Textsorten bietet das Buch „Textsorten im Deutschen: linguistische Analysen aus sprachdidaktischer Sicht" von Christian Fandrych und Maria Thurmair (2011). Die Autoren haben die spezifische Anwendung sprachlicher Mittel in zwanzig Textsorten (darunter die Textsorte „Audioguide“) der deutschen Gegenwartssprache analysiert und so einen umfassenden Einblick in unterschiedlichste Formen und Bereiche der heutigen Kommunikation ermöglicht. In diesem Beitrag setzten sich die Autoren mit der Textsorte „Audioguide“ nicht nur aus einer linguistischen, sondern vielmehr aus einer sprachdidaktischen Perspektive auseinander.

Unter dem Titel „Kunst besprechen und vermitteln: $\mathrm{Zu}$ sprachlichen Strategien von Audioguides“" ist im Jahre 2015 eine weitere Abhandlung von denselben Autoren erschienen. Im Mittelpunkt ihrer Untersuchung stehen hauptsächlich die Beziehung zwischen Kunstwerk und Text einerseits sowie die Rezeption des Audioguide-Textes durch den Hörer andererseits. Sie betonen, dass Audioguides konstatierend-assertierend auf ein 
Audioguides. Oder „Mit Ohren sehen“!

Zur Übersetzung der Textsorte „Audioguide“ als Texte der Kunstkommunikation am Beispiel des Textes „Früher Lagerhalle, heute Moschee“6

Dr. Mohamed Nasef

مجلة وادي النيل للاراسات والبحوث الإنسانية والاجتماعية والتربوية (مجلة علمية محكمة)

bestimmtes Kunstobjekt orientiertes Wissen bieten. Audioguides können im Allgemeinen für Kunstkommunikation angepasste sprachliche Teilhandlungen mit eigenen Funktionen aufzeigen.

Der Aufsatz „Audioguides: Die Inszenierung von Kunst im Hörtext" diskutiert, welche sprachlichen Strategien in den Hörtexten von Audioguides verwendet werden sollen. Hier steht das Kunsterlebnis der Besucher im Mittelpunkt. Dabei wird besonders auf die Formen der Bilderschließung eingegangen und eine Typologie der vorzufindenden Strategien aufgestellt (vgl. Fandrych/ Thurmair 2016).

Aus einer Übersetzungsperspektive ist ein Beitrag von Ema Bašić (2020) unter dem Titel „Übersetzung von Audioguides am Beispiel des Kunsthistorischen Museums Wien und Neandertalermuseums in Krapina" erschienen. Dabei hat die Autorin anhand von Textbeispielen den Audioguide des Kunstmuseums Wien und des Neandertalermuseums und seine jeweilige Übersetzung in Bosnisch/Kroatisch/Serbisch und Deutsch verglichen und analysiert. Ihre Kriterien zur Analyse waren die Übersetzungsstrategien und Merkmale der Sprache in der Kunstkommunikation. Weiterhin verblieb dieser Aufsatz im Rahmen des Übersetzungsvergleiches zwischen dem Deutschen und den oben erwähnten Sprachen.

Vor dem Hintergrund der oben dargestellten Beiträge ergibt sich, dass das Phänomen „Audioguide“ als ein Teil der Vermittlungskommunikation im großen Bereich der Kunstkommunikation bereits aus verschiedenen Perspektiven behandelt wurde. Eine Studie, die sich speziell mit dem Thema „Übersetzung von Audioguide-Texten zwischen Arabisch und Deutsch in Ägypten“ befasst, ist jedoch nicht zu finden. Dieser Beitrag wird sich in seinem Kern auf diese Problematik konzentrieren. Im Folgenden soll aber zuerst auf die Kommunikation des Audioguides im Bereich der Kunstkommunikation eingegangen werden. Dabei wird die 
مجلة وادي النيل للاراسات والبحوث الإنسانية والاجتماعية والتربوية (مجلة علمية محكمة)

(ISSN : 2536 - 9555)

Beziehung zwischen Kunstkommunikation bzw. Text und Rezeptionssituation betrachtet.

\section{Kommunikationssituation}

Da Audioguides zum Bereich der Kunstkommunikation gehören, wird dieser Begriff näher beschrieben. Hausendorf (2011) zufolge kann der Begriff „Kunstkommunikation“ nicht auf „Kommunikation über Kunst" reduziert werden. Der Begriff „Kunstkommunikation“ weist auf eine in der Gesellschaft stattgefundene Kommunikation mit und durch Kunstwerke hin (vgl. Hausendorf 2011:510). Auf diese Weise hängt Kunstkommunikation eng mit der Rezeption von Kunstgeschehnissen zusammen. In diesem Kontext lassen sich zwei Typen von Kommunikation unterscheiden: Der erste Typ ist die Vorauskommunikation. Damit ist die Lektüre eines Ausstellungskatalogs zur Vorbereitung auf den Besuch einer Ausstellung gemeint. Der zweite Typ ist die simultane Kommunikation, wie wir sie bei Audioguides vorfinden. Hier läuft die Rezeption durch den Besucher (bzw. Hörer) gleichzeitig mit der Rezeption der Kunstgeschehnisse ab. Zusammengenommen geht es bei Audioguides also um echte, gleichzeitige Kommunikation im Gegensatz zur Vorauskommunikation. Deswegen werden Audioguides durch eine starke Relation zwischen Text und Kunstwerk charakterisiert (vgl. Fandrych/Thurmair 2011: 73).

Nun setzen wir uns näher mit der Rezeptionssituation auseinander. Zwar sind Audioguides schriftlich formuliert, aber sie werden von einem Sprecher realisiert: Sie sind also üblicherweise vorformuliert. Als Voraussetzung zur Verwirklichung dieser Textsorte gilt die Regel, dass der Rezipient die Audioguides nur als Hörer aufnehmen kann. Deshalb sind sie von kurzer Dauer und nur der Rezipient entscheidet über die Bedingungen der Rezeption (also wann und wie oft er die Texte hört). 
Audioguides. Oder „Mit Ohren sehen“!

Zur Übersetzung der Textsorte „Audioguide“ als Texte der Kunstkommunikation am Beispiel des Textes „Früher Lagerhalle, heute Moschee“6

Dr. Mohamed Nasef

مجلة وادي النيل للاراسات والبحوث الإنسانية والاجتماعية والتربوية (مجلة علمية محكمة)

Was die Rezeption von Hörtexten allgemein und insbesondere die von Audioguides anbelangt, sollen diese gesprochenen Hörtexte spezielle Voraussetzungen bezüglich ihrer Rezeption erfüllen: Hörtexte bereiten Schwierigkeiten in der Rezeption, da sie - im Gegensatz zu schriftlichen Texten - größtenteils kurz und flüchtig sind. Aus diesem Grund sind sie stärker kontextgebunden, das heißt das Verständnis von Audioguides verlässt sich im Grunde auf dem Rezeptionskontext. Dabei ist es von besonderer Bedeutung, dass der Hörer vor dem Hören eine klare Annahme über den Kontext aufstellt. Diese sogenannte Vorerwartung erleichtert die Aufnahme von Hörtexten. Der Hörer einer AudioFührung kann also ganz spezifische Hypothesen über den Inhalt des Hörtextes aufstellen, die sich auf sein Vorwissen und seine Vorerfahrung mit dem Textmuster „Kunstführung“ gründen: Sowohl Thema und Struktur als auch sprachliche Handlungen kann er bereit im Vorfeld vermuten. Angesichts der Hörstile bzw. Hörstrategien bieten sich drei Hörstile an: Der globale, der selektive und der detaillierte Hörstil. Beim globalen Hören versucht der Hörer, sich eine allgemeine Vorstellung vom Inhalt des Textes zu verschaffen. Selektive Hörer konzentrieren sich auf ausgewählte Informationen des Textes. Beim detaillierten Hörstil rezipiert der Hörer den Text Satz für Satz, um die detaillierten Ideen des Textes zu erfassen. Audioguides sind eine Textsorte, die detailliert begriffen werden soll. In den meisten Fällen bevorzugen die Hörer bzw. Besucher den selektiven Hörstil. Dies steht der Intention des Produzenten entgegen, welcher an den detaillierten Hörstil der Hörer/ Besucher des Kunstwerks appelliert hat (vgl. Fandrych/Thurmair 2011: 74f.).

Was die Kunstkommunikation betrifft, so sind folgende fünf Teilhandlungen mit spezifischen Funktionen in Anlehnung an Hausendorf (2011) zu unterscheiden: a) Beschreiben: was gibt es zu sehen, hören, tasten ...? b) Deuten: was steckt dahinter? c) Erläutern: was weiß man darüber? d) Bewerten: was ist davon zu 
(ISSN : 2536 - 9555)

halten? e) Bezugnehmen: worum geht es? Im Falle von Audioguides wird besonders dem Erläutern eine wichtige Bedeutung zugewiesen, da es sich bei ihnen um informative Texte handelt, welche Fachwissen reflektieren. Dies kann durch die didaktische Intention des Textes realisiert werden. In anderen Situationen sind die einzelnen Teilhandlungen nach Hausendorf (2011) weitgehend miteinander sowie teilweise auch mit weiteren sprachlichen Handlungen verknüpft.

\section{Aufbau von Audioguides}

Nach dem Motto „Mit den Ohren sehen” werden Audioguides im musealen Umfeld bzw. in Kulturprojekten eingesetzt. Dabei handelt es sich um akustisch eingerichtete Hörgeräte für die Verwendung bei Ausstellungsbesucher-Rundgängen. Mit diesen Audiogeräten kann man durch Nummerneingabe zu bestimmten Objekten inhaltliche und darauf gespeicherte Informationen abrufen. Der Textinhalt der Audioguides ist so eingerichtet, dass die Informationen bildhaft und nutzergerecht vermittelt werden. Laut Dech (2004) soll der Audioguide-Text aus Begrüßung und Einführung bestehen. Im Anschluss daran sollen weiterführend Hintergrund, Zusatzinformationen sowie ein Abschlusstext folgen (vgl. Dech 2004: 32). Hier ist es wichtig, zu erwähnen, dass die von Audioguides rezipierten Informationen allgemeinverständlich sein müssen. Noch dazu muss der Textinhalt von Audioguides wissenschaftlichen Ansprüchen genügen. Audioguides werden verwendet, um genaue Details über Exponate zu vermitteln. Dabei gilt als entscheidender Faktor, dass auditive Informationen eine Bildbeschreibung präsentieren, mit deren Unterstützung der Besucher geleitet werden kann (vgl. Schultze/Buhl 2012: 28). Um dies zu realisieren, spricht Dillmann (2012) von bestimmten Regeln, die zu befolgen sind: Hinsichtlich der Textsätze sollen Audioguide-Texte nicht lang sein und begrenzt gehalten werden. Für jeden Satz ist eine einzelne Information ausreichend. Die Verben der Sätze sollen nach Möglichkeit am Anfang des Satzes stehen. Falls zweistellige Verben vorkommen, sollen diese lieber 
Audioguides. Oder „Mit Ohren sehen“!

Zur Übersetzung der Textsorte „Audioguide“ als Texte der Kunstkommunikation am Beispiel des Textes „Früher Lagerhalle, heute Moschee“6

Dr. Mohamed Nasef

مجلة وادي النيل للاراسات والبحوث الإنسانية والاجتماعية والتربوية (مجلة علمية محكمة)

zusammengesetzt stehen. Die zentralen Informationen sollen im Vordergrund platziert werden. Für wichtig gehaltene Begriffe und Namen sollen wiederholt werden. Mit Synonymen soll vorsichtig umgegangen werden. Auf Fachbegriffe und Fremdwörter soll laut Dillmann (2012) verzichtet werden. Falls notwendig sollen diese mit Erklärungen und Interpretationen versehen werden. Verbalstile eignen sich besser als Nominalstile. Dillmann bevorzugt die Verwendung der Aktivform statt des Passivs. Adjektive sollen auftreten, ein Text soll jedoch nicht über die Maßen. Diese Regeln sollten nicht aus den Augen verloren werden, da die Ausstellungsbesucher im Nachhinein keine Möglichkeit mehr haben, Einwände vorzubringen.

\section{Sprachliche Merkmale von Audioguides}

Die Textsorte Audioguide ist durch verschiedene sprachliche Merkmale gekennzeichnet, die im Folgenden in Anlehnung an Fandrych/Thurmair (2011) näher erläutert werden:

1. Herstellung von Adressatenbezug durch Verwendung des Pronomens „wir“. Aus den vorigen Ausführungen ist deutlich geworden, dass Audioguide-Texte didaktische Ziele verfolgen. Dies wird auf der sprachlichen Ebene durch unterschiedliche Formen realisiert. Solche Formen dienen zum Produzieren eines direkten Adressatenbezugs durch den Einsatz des Pronomens „Wir“. Das Pronomen kann je nach Kontext auf verschiedene Gruppen hinweisen, entweder explizit oder impliziert (z. B. Gruppe der Experten oder Fachleute).

\section{Herstellung von Adressatenbezug durch Fragen}

Die Überleitung in Frageform wird in Audioguide-Texten häufig genutzt, um einen direkten Bezug zum Adressaten zu schaffen. Der Besucher wird so direkt angesprochen. Die Fragen motivierten ihn, am weiteren Geschehen aktiv und interessiert 
مجلة وادي النيل للاراسات والبحوث الإنسانية والاجتماعية والتربوية (مجلة علمية محكمة)

(ISSN : 2536 - 9555)

teilnehmen $\mathrm{zu}$ können. Außerdem steigern die Fragen die Motivation des Adressaten am Verstehen des Textes; wie im folgenden Beispiel aus Max Liebermann „Münchener Biergarten“: [Kommen Sie doch näher, setzen Sie sich nieder auf ein Glas Bier! Oder wollen Sie noch ein wenig stehen bleiben und die Leute betrachten? Was sehen Sie denn?]

\section{Räumliche Orientierung}

Um den Kunstgegenstand so genau wie möglich darzustellen, spielt die räumliche Orientierung bei der Audioguide-Führung eine zentrale Rolle. Hier verfolgt die Bildbeschreibung das Ziel, den Hörer eine Vorstellung von der Rezeptionssituation entwickeln zu lassen. Die Raumorientierung erfolgt im Audioguide-Text durch bestimmte Lokalangaben wie „vorne“, „rechts“, „links“ usw. Fandrych/Thurmair (2011) geben weitere Beispiele an, die sich auf zweidimensionale Bildflächen beziehen, etwa ,: [... quer ins Bild ragt, rechts zu sehen]. Sie führen zudem weitere Beispiele für den dreidimensionalen Raum an, wie „davor/ in dem Vordergrund“, ,in die tief verschattete Vordergrundszene“" usw.

\section{Negation}

Mit den Texten von Audioguides sind seitens des Hörers bestimmte Erwartungen verbunden. Durch diese Erwartungen wird der Adressatenbezug hergestellt. Solche Erwartungen können entweder angenommen und bestätigt oder revidiert bzw. gestoppt werden. Man spricht erst von einem „Erwartungsstopp“, wenn Negationselemente auftauchen. Da durch Negation eine bestehende Erwartung nicht erfüllt wird, erlebt der Hörer eine Erwartungslenkung. Dadurch wird seine Aufmerksamkeit erregt und er wird dazu angeregt, dem weiteren Text genau und detailliert zu folgen. Als Beispiel für die Negation führen Fandrych/Thurmair (2011) folgenden Satz an: [Auf diesem Bild zeigt uns Caspar David Friedrich keine grünende und blühende Natur].

\section{Attribute}

Attribute sind relevante sprachliche und syntaktische Mittel, die eine nähere Bestimmung nominaler Satzglieder markieren. 
Audioguides. Oder „Mit Ohren sehen“!

Zur Übersetzung der Textsorte „Audioguide“ als Texte der Kunstkommunikation am Beispiel des Textes „Früher Lagerhalle, heute Moschee“6

Dr. Mohamed Nasef

مجلة وادي النيل للاراسات والبحوث الإنسانية والاجتماعية والتربوية (مجلة علمية محكمة)

Wesentliche Aufgabe der Attribute ist es, die Bezugsausdrücke zu modifizieren, die mit entsprechenden Substantiven „bezeichneten Referenzobjekte“ zu beschreiben und exakt zu kennzeichnen (vgl. Thurmair 2018: 415). Sie können außerdem als Hilfsmittel beim Identifizieren der fokussierten Objekte auf dem Bild dienen. In diesem Zusammenhang erwähnen Fandrych/Thurmair (2011), dass in Audioguide-Texten eine Vielzahl von prä- und postnuklearen Attributen sowie Attributtypen erscheinen. Im Allgemeinen enthalten die Texte der Audioguides ,viele kategoriale Attribute mit wiederum anderer funktionaler Spezifik. [...] Attributive Adjektive werden eingesetzt, die als evaluative Adjektive der Wertung dienen“ (Thurmair 2015: 376). In diesem Fall beziehen sie sich auf die Szene, die das Kunstwerk präsentiert, wie z. B.: [Ein heißer Wind rauscht durch das Schiff, schwüle Mittagshitze lastet über dem Gebüsch]. Sie können aber auch Wie im folgenden Beispiel mit dem Status als Kunstwerk verknüpft werden:[Allein wie das Blut im Grunde in Adern gezeigt wird, das ist eine ganz wunderbarer durchpulste, lebendige Körperlichkeit, das ist eine große Meisterschaft] (ebd.: 376).

\section{Kennzeichen der Mündlichkeit}

Die Mündlichkeit ist ein wichtiges Merkmal, das AudioguideTexte kennzeichnet, da diese Texte zwar schriftlich konzipiert, aber mündlich realisiert werden. Diese Mündlichkeit hat zwei Formen: Die sind zum Einen syntaktische Kennzeichen, d.h. sie umfassen alles, was die Wortstellung angeht, und zum anderen lexikalische Kennzeichen, welche den Einsatz von Adverbien bzw. Partikeln wie da, also betreffen. Die lexikalischen Kennzeichen dienen der Fortführung eines Themas und sind eine charakteristische Eigenschaft des mündlichen Erzählens, welche das Erzählen lebendiger und interessanter macht. 
(ISSN : 2536 - 9555)

\section{Audioguide als audiomedialer Texttyp}

Weil Audioguides sich auf außersprachliche Medien beziehen, gehören sie $\mathrm{zu}$ den audiomedialen Texttypen. Als Übersetzungsmethoden für diesen Texttyp schlägt Reiß vor, beim Übersetzen medien- bzw. verbundgerecht (suppletorisch) vorzugehen (vgl. Reiß 1976: 23). Jedoch wird hier die Ansicht vertreten, dass die von Reiß vorgeschlagene Übersetzungsmethode nicht für jeden Texttyp geeignet ist, da die Interpretation des Textes beim Übersetzen als rein subjektive Handlung gilt und sich die Interpretation des Textes dadurch ändern kann. In der Übersetzungswissenschaft hat man den Begriff ,audiomedial“ durch die Bezeichnung „multimedial“ ersetzt. Dennoch finden sich Textsorten, die zwar schriftlich festgehalten sind, aber in gesprochener Form das Ohr des Rezipienten erreichen. Diese sind nicht multimedial. In diesem Fall geht es um Texte, die absichtlich zum Sprechen eingesetzt werden. Aufgrund dessen können diese Texttypen als audiomedial markiert werden. $\mathrm{Zu}$ ihnen gehören wissenschaftliche Vorträge, Bibeltextstellen und auch AudioguideTexte. Sie erreichen den Empfänger akustisch über die menschliche Stimme (vgl. Snell-Hornby 1999: 273).

\section{Korpus und die methodische Vorgehensweise}

Im Folgenden wird der Text näher betrachtet, welcher die Basis für den vorliegenden Beitrag bildet. Darauf folgend wird die methodische Vorgehensweise näher erläutert.

\subsection{Textauswahl}

Grundlage der vorliegenden Untersuchung ist der Audioguide „Früher Lagerhalle, heute Moschee“. Er wurde in der Reihe „Religiöse Orte in Göttingen“ der Georg Augustin Universität Göttingen im Rahmen des Audioguide-Projekts ${ }^{3}$ „,Religionen in Göttingen" veröffentlicht. Da dieser Audioguide nicht von

3. Mehr zu diesem Projekt finden Sie unter: https://www.unigoettingen.de/de/615632.html. 
Audioguides. Oder „Mit Ohren sehen“!

Zur Übersetzung der Textsorte „Audioguide“ als Texte der Kunstkommunikation am Beispiel des Textes „Früher Lagerhalle, heute Moschee“6

Dr. Mohamed Nasef

مجلة وادي النيل للاراسات والبحوث الإنسانية والاجتماعية والتربوية (مجلة علمية محكمة)

organisierten Anbietern bereitgestellt wird, gehört er zum Typ „,nutzergenerierte Audioguides“.

Der Audioguide fokussiert auf einen religiösen Ort, nämlich die al-Taqwa-Moschee in Göttingen. Diese Moschee war ehemals eine Lagerhalle. Der Verfasser hat diesen Text insbesondere ausgewählt, weil im Mittelpunkt ein Ort steht, der auf die eine oder andere Weise einen religiösen Stellenwert hat. Wenn man das Wort „Moschee“ hört, fällt sofort auf, dass dieser Ort ursprünglich religiös war. Das Neue an dem Text ist, dass einem heutigen religiösen Ort früher eine nicht religiöse Bedeutung innewohnte. Statt Güterzügen herrscht heute ein engagiertes Gemeindeleben im Gebäude. Im Audioguide wird beschrieben, wie eine ehemalige Lagerhalle in eine Moschee verwandelt wird und wie dort das islamische Freitagsgebet stattfindet.

\subsection{Methodische Vorgehensweise}

Die Untersuchung gliedert sich in die folgenden Phasen:

1. Phase: Die schriftliche Wiedergabe des gehörten Textes und seine Übersetzung ins Arabische.

2. Phase: Diskussion über die arabische Übersetzung.

3. Phase: Analyse des Audioguides nach den Kriterien der sprachlichen Merkmale.

1. Phase: Die schriftliche Wiedergabe4 des Audioguides „Früher Lagerhalle, heute Moschee“" und seine Übersetzung ins Arabische

Göttinger al-Taqwa Moschee in der Güterbahnhofstraße.

4. Den Audioguide-Text meines Korpus habe ich als Vorbereitung für die Analyse bzw. Diskussion sowie auch die Übersetzung in eine schriftliche Version umgewandelt. Das heißt: Die Sätze werden hier schriftlich wiedergegeben. 
(1) Wir befinden uns in einem weiten lichtdurchfluteten Raum.

$$
\text { من هذه القاعة الو اسعة المضيئة أتحدث إليكم }
$$

(2) Unter unseren Füßen weicher dunkelroter Teppich.

$$
\text { أرضيتها مفروشة بسجاد ناعم لونه احمر غامق }
$$

(3) Unsere Schuhe haben wir am Eingang ausgezogen und in ein Regal einsortiert.

$$
\text { قمنا بخلع أحذيتنا عند المدخل ووضعناها في الرف المخصص للأحذية }
$$

(4) Zu unserer Linken befindet sich die Minbar ${ }^{5}$.

$$
\text { على جهة الثمال يوجد المنبر }
$$

(5) Sie ähnelt einer kurzen hölzernen Treppe.

$$
\text { و هوجسم يثبه السلالم الخثبية القصبرة }
$$

(6) Auf ihr wird freitags gepredigt. Daneben steht Pult und Ventilator.

$$
\text { يقف عليه الأمام أثناء خطبة الجمعة و على جانبيه يوجد منصة ومروحة }
$$

(7) Es ist ruhig.

$$
\text { يخيم الهذو على المكان }
$$

(8) Nur ab und an das Rauschen eines vorbeifahrenden Zuges und das Lachen eines spielenden Kindes.

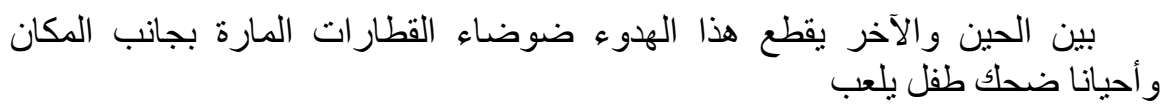

5. Kanzel der Moschee, über einer Treppe erhöht aufgestellter Predigtstuhl in einer Moschee. 
مجلة وادي النيل للاراسات والبحوث الإنسانية والاجتماعية والتربوية (مجلة علمية محكمة)

(9) Über uns schweben Kronleuchter.

$$
\text { وفوق رؤوسنا يتأرجح ضوء الثريا }
$$

(10) Ein dichter Vorhang teilt den Raum in zwei.

$$
\text { وستارة سميكة تفصل القاعة إلى جز عين }
$$

(11) An eine Wand haben sich zwei Stühle verirrt.

$$
\text { تم وضع كرسيين بجانب الحائط }
$$

(12) Große bodentiefe Fenster erstrecken sich $\mathrm{zu}$ unserer Rechten.

$$
\text { شباك كبير يصل إلى الأرض يمتد على الناحية اليمنى }
$$

(13) Gemusterte Milchglasfolien schützen vor neugierigen Blicken.

$$
\text { زجاج رقيق منقوش لبني اللون يمنع نظر ات المتطفلين }
$$

(14) Von außen sind Zahlen zu erkennen, deren Farben bereits abblättert.

$$
\text { ومن الخارج يُرى العديد من الأرقام التي تقشرت ألو انها بالفعل }
$$

(15) Unter ihnen befindet sich eine lange Laderampe.

$$
\text { منصة تحميل البضائع بالقرب منها }
$$


(ISSN : 2536 - 9555)

(16) Nur diese Zahlen und die Umgebung verraten uns, wofür dieser Raum früher einmal genutzt wurde.

الأرقام المكتوبة والمنطقة المحيطة تكثف لنا فيما كانت تستخدم هذه القاعة قديما

(17) Direkt hinter dem Gebäude verlaufen die Bahnschienen.

تمتد خلف هذه البناية خطوط السكة الحديد

(18) Und genau diese Bahnschienen verliefen früher einmal bis an die Laderampe direkt unter den Fenstern.

تمتد قضبان السكة الحديد مباشرة حتى منصة تحميل البضائع خلف شباك القاعة

(19) Wir befinden uns in einer ehemaligen Lagerhalle der Bundesbahn.

ها نحن نتو اجد في إحدى المخازن السابقة التابع لسكك حديد المانيا الاتحادية

(20) Ein wenig nördlich vom Bahnhof gelegen, lagerten hier jahrelang Güter.

على مدار سنين طويلة كانت البضائع تُخزن فى الجهة الثمالية من محطة القطار

(21) Nachdem die Halle ausgedient hatte, stand sie für 30 Jahre leer.

$$
\text { وبعد أن خرجت هذه القاعة من الخدمة ظلت لمدة • ب عام فارغة }
$$

(22) 2010 erwarb der muslimische Kulturverein diese Halle und das Bürogebäude.

وفى عام • ـ ا ب امتلكت جمعية الثقافة الإسلامية هذه القاعة وكذلك مبنى المكاتب 
مجلة وادي النيل للاراسات والبحوث الإنسانية والاجتماعية والتربوية (مجلة علمية محكمة)

(23) Die muslimische Gemeinde der al-Taqwa Moschee renovierte die Gebäude und gestaltete sie um. Heute finden hier Gebete statt.

قامت الر ابطة الإسلامية التابعة لمسجد التقوى بترميم هذه القاعة و إعادة تقسيمها

$$
\text { وأصبحت اليوم مكانًا لإقامة الصاملة الصلابعة }
$$

(24) Am Wochenende auch Arabisch- und Islamunterricht.

$$
\text { وفي نهاية الأسبوع تصبح مكان لتعليم اللغة العربية و العلوم الإسلامية }
$$

(25) Im Untergeschoss gibt es die Möglichkeit für bis zu 300 Personen zu kochen und Feste zu feiern.

الطعام والاحتفال بالأعياد السفي لعدد كبيرمن الزائرين يصل إلى . . شخص يمكنهم إعداد

(26) Die religiösen Feste aber auch Hochzeiten.

$$
\text { أيضا الاحتفال بالأعياد الدينية والأعراس }
$$

(27) Dann wandelt sich der jetzt so ruhige Gebetsraum in eine Kinderspielwiese mit weichem Teppich.

وسر عان ما تتحول هذه القاعة إلى ساحة العاب للأطفال ذات أرضية طرية

(28) Der Raum zeichnet sich durch seine Schlichtheit aus.

$$
\text { حيث تتميز هذه القاعة ببساطتها }
$$

(29) Die Ausstattung beschränkt sich auf das Notwendigste.

$$
\text { فهي تقتصر فقط على الأشياء الضرورية }
$$


(ISSN : 2536 - 9555)

(31) Auf diesen Wunsch reagierte die Gemeinde beispielsweise mit der Gestaltung der Eingangstür.

$$
\text { طبقا لذلك قامت الر ابطة على سبيل المثال بتصميم باب المدخل }
$$

(32) Mit Schnitzereien und kleinen Goldverzierungen wirkt sie fast antik.

$$
\text { ومن خلال بعض النقوش و الزخارف الذهبية البسيطة بَدَت وكأنها تحفة معمارية }
$$

(33) Dabei erwarb die Gemeinde die Tür erst kürzlich für wenig Geld von einem Schreiner aus Marokko.

ومن هنا قامت الر ابطة بطلاء الباب منذ فترة بدر اهم معدودة تبر ع بها نجار مغربي

(34) Den arabischen Schriftzug fertigte eine soziale Einrichtung in Göttingen an.

جوتنجن أما رقم الحروف العربية فقد تكفلت بها مؤسسة الضمان الاجتماعي في مدينة

(35) Damit vereint diese Tür sowohl den Wunsch der Gemeinde nach Ästhetik als auch Imam „Kanjus“ theologische Anforderungen an eine Moschee sowie seine Überzeugung vorhandenes Geld eher für Bedürftige auszugeben.

وبذلك تم التوفيق بين رغبة الجمعية فى التجميل وبين قناعات الامام فى ضرورة بساطة المكان و انفاق نقود التبر عات على على المحتاجين

(36) Heute werden in dem schlichten Raum keine Güter mehr gelagert.

$$
\text { لم تعد هذه القاعة المتو اضعة فى الوقت الر اهن مخزنًا للبضائع }
$$


Audioguides. Oder „Mit Ohren sehen“!

Zur Übersetzung der Textsorte „Audioguide“ als Texte der Kunstkommunikation am Beispiel des Textes „Früher Lagerhalle, heute Moschee“6

Dr. Mohamed Nasef

مجلة وادي التيل للاراسات والبحوث الإنسانية والاجتماعية والتربوية (مجلة علمية محكمة)

(37) Die Menschen kommen an diesen Ort, um zu beten, um zu lernen und um gemeinsam Feste zu feiern.

و إنما تحولت إلى مكان متميز يفد إليه الناس للصلاة والتعليم وكذلك الاحتفال بالأعياد

(38) Für die Waren war dieser Raum damals nur eine Zwischenstation.

$$
\text { حيث كانت هذه القاعة في السابق عبارة عن مركز تخزين مؤقت للبضائع }
$$

(39) Durch die Göttinger al-Taqwa Gemeinde hingegen wandelte er sich in ein Ort des Verweilens und des Glaubens.

$$
\text { إلا أنها تحولت اليوم بفضل رابطة مسجد التقوى بمدينة جوتتجن إلى مكان للعبادة }
$$

\section{Phase: Diskussion über die arabische Übersetzung}

Dieser Teil ist der Darstellung von den Ergebnissen der Analyse gewidmet. Hier wird die arabische Wiedergabe des Audioguide-Textes thematisiert. Dabei wird ein Überblick über die wichtigsten Ansatzpunkte bei der Übersetzung geworfen. Als erster Schritt wurde der auf Deutsch gesprochene Audioguide-Hörtext vom Verfasser dieses Aufsatzes schriftlich wiedergegeben und ins Arabische übersetzt. Der Ausgangssprache-Hörtext (AS-Hörtext) gliedert sich in mehrere akustische Episoden, wie im Folgenden gezeigt wird:

Der Audioguide-Hörtext beginnt mit dem Einführungssatz: (1) Wir befinden uns in einem weiten Raum. Obwohl das Arabische bei der Satzbildung stark zur Verbalsatzform neigt, d. h. die normale Verb-Subjekt-Objekt (VSO)-Wortstellung, wurde der Satzanfang in der arabischen Version durch die unterstrichene Präpositionalphrase من هذه القاعة الو اسعة المضيئة أتحدث إليكم ersetzt, um 
(ISSN : 2536 - 9555)

die Aufmerksamkeit des Zielsprachen (ZS)-Hörers zu gewährleisten. Hier spricht man von der Bildbeschreibung der Einrichtung durch Methoden bzw. Mittel des AS-Textes.

Die Attribute im Satz Nr. (2) Unter unseren Füßen weicher dunkelroter Teppich sind wichtige syntaktische Mittel für die Beschreibung des Sachverhalts. Sie folgen dem Ziel, ihre Bezugsausdrücke zu modifizieren. In diesem Satz tragen die Attribute dazu bei, die in Fokus genommenen Objekte in der Kultureinrichtung (weicher dunkelroter Teppich) zu identifizieren. Bei der Wiedergabe im Arabischen musste die Präpositionalphrase unter unseren Füßen durch das Possessivpronomen أرضيتها ersetzt werden, da die wörtliche Übersetzung تحت أقدامنا aus ästhetischen Gründen nicht akzeptabel ist. Die Adjektive sind jedoch wörtlich problemlos سجاد ناعم لونه أحمر غامق übertragen worden.

Die Widergabe des AS-Satz Nr. (4) Zu unserer Linken befindet sich die Minbar durch den ZS-Satz: على جهة الثمال يوجد المنبر reflektiert ein Bild des fiktiven Sprechers, der „eine Perspektive aus dem Bild an[nimmt] und [...] den Rezipienten direkt an[spricht]" (Fandrych/Thurmair 2016: 388). Er schildert dabei das Kunststück (Satz Nr. 5) Sie ähnelt einer kurzen hölzernen Treppe unter dem Motto „das Bild zum Leben zu erwecken“ (ebd.). Durch die arabische Entsprechung وهوجسم يشبه السلالم الخشبية entsteht ein Dialog mit dem Hörer, welcher den Hörer mit Handlungsaufforderungen und -alternativen konfrontiert (vgl. ebd.). Als Beschreibung für die Hauptaufgabe des Lexems Minbar gelten die beiden anschließenden Sätze: (6) Auf ihr wird freitags gepredigt. Daneben steht Pult und Ventilator. Der zweite Teil des Satzes wurde durch einen ZS-Attributsatz bzw. asyndetischen Relativsatz ersetzt. Die AS-Sätze kommen im Arabischen wie folgt

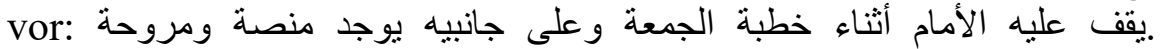
Obwohl das aus dem Arabischen entliehene Wort Mimbar in der deutschen Sprache schon integriert ist, gibt dieser Satz dem Rezipienten, der eventuelle Wissensdefizite in diesem Zusammenhang hat, in beiden Sprachen auf interessante Weise genügend Informationen. Dies zeigt die Fähigkeit des Sprechers, 
Audioguides. Oder „Mit Ohren sehen“!

Zur Übersetzung der Textsorte „Audioguide“ als Texte der Kunstkommunikation am Beispiel des Textes „Früher Lagerhalle, heute Moschee“6

Dr. Mohamed Nasef

مجلة وادي التيل للاراسات والبحوث الإنسانية والاجتماعية والتربوية (مجلة علمية محكمة)

die Kultureinrichtung nicht nur zu beschreiben, sondern auch zu erläutern.

Der AS-Satz Nr. (7) Es ist ruhig schildert die Lage in der Einrichtung. Hier übernimmt der Sprecher des Audioguides die Rolle als ,kompetenter Betrachter“" (Fandrych/Thumair 2016: 383) und setzt das Bild in Wörter um, um dem Hörer ein volles Erlebnis der Einrichtung $\mathrm{zu}$ ermöglichen. Als angemessenes arabisches Äquivalent für den oben angeführten Satz gilt das خيم Funktionsverbgefüge bzw. die metaphorische Kollokation im Sinne von „Die Ruhe herrscht“. Dafür brauchte der Übersetzer eine translatorische Kompetenz, um die kommunikative Funktion und ästhetische Wirkung der Zielsprache zu erhalten.

Die Wiedergabe des teilnegierten Satzes (32) Mit Schnitzereien ومن خلال بعض النقوش وبدون أى زخارف بدت وكأنها تحفة معمارية zentrale Rolle der Negationselementen innerhalb des AudioguideTextes. Durch die Struktur und kein Goldverzierung werden gewisse Erwartungen zuerst angenommen und dann durch die Negation gestoppt. Gemäß der wörtlichen Übersetzung sollten die Negationspartikel als ليس oder Vvorkommen, was die Struktur des ZS-Satz nicht zulässt. Aus einer stilistischen Perspektive wurde die Negationspartikel durch ZS-Präposition بدون (dt.: ohne), die im Arabischen für solchen Negationszweck verwendet, ersetzt. Das führt ebenfalls dazu, dass der arabische Satz harmonisch fließt.

\section{Phase: Die Analyse des Audioguides nach den Kriterien der sprachlichen Merkmale}

Die vorher erwähnten sprachlichen Merkmale (im Punkt 6) spiegeln sich in dem gesamten Audioguide wieder. Im Folgenden 
(ISSN : 2536 - 9555)

sollen die im Audioguide-Text vorherrschenden Merkmale anhand einiger Beispiele aus dem Audioguide-Text illustriert werden.

Durch die Verwendung des Pronomens „wir“ wird ein Adressenbezug hergestellt. Mit dem ,wir“ wird eine Gruppe aus Sprecher und Angesprochenen gebildet, z. B. in:

(1) Wir befinden uns in einem weiten Raum,

(3) Unsere Schuhen haben wir am Eingang ausgezogen und in ein Regal einsortiert, (19) Wir befinden uns in einer ehemaligen Lagerhalle der Bundesbahn.

In diesen Beispielen wird das ,wir“ eingesetzt, um Sprecher und Adressaten auch im selben Bild zu positionieren.

Die räumliche Orientierung ist bei einem Audioguide sehr wichtig, weil durch sie die Wege und Orte so beschrieben werden, dass der Hörer sich das Bild einfacher vorstellen kann. Bei der Beschreibung von Orten in Audioguides sollte einer langsamen und deutlichen Aussprache Beachtung geschenkt werden. Dies erscheint ganz deutlich in den folgenden Beispielen:

(15) Unter ihnen befindet sich eine lange Laderampe.

(17) Direkt hinter dem Gebäude verlaufen die Bahnschienen.

(20) Ein wenig nördlich vom Bahnhof gelegen, lagerten hier jahrelang Güter. (25) Im Untergeschoss gibt es die Möglichkeit für bis zu 300 Personen zu kochen und Feste zu feiern.

Im Audioguide ist eine Bezugnahme auf unterstellte Erwartungen durch die Verwendung von Negation zu bemerken. Die Negationsartikel zeigen erkennbaren Adressatenbezug auf. Sie „zielen auf die Hörererwartung insofern sie, [...] grundsätzlich eine vermutete Erwartung bezeichnen" (Fandrych/Thurmeir 2011: 85). Im vorliegenden Audioguide-Text kommen oft Negationspartikel vor, mit denen die angenommene Erwartung nicht erfüllt ist. Hierfür sind Beispiele aus dem Text: 
Audioguides. Oder „Mit Ohren sehen“!

Zur Übersetzung der Textsorte „Audioguide“ als Texte der Kunstkommunikation am Beispiel des Textes „Früher Lagerhalle, heute Moschee“6

Dr. Mohamed Nasef

مجلة وادي النيل للاراسات والبحوث الإنسانية والاجتماعية والتربوية (مجلة علمية محكمة)

(32) Mit Schnitzereien und kleinen Goldverzierungen wirkt sie fast antik.

(36) Heute werden in dem schlichten Raum keine Güter mehr ausgelagert.

Da Audioguide-Texte nur mündlich wiedergegeben werden, werden bei ihrer Erstellung die Merkmale der Schriftlichkeit vor Augen gehalten. Deswegen werden bei einigen Audioguides ein paar Textstellen geschoben. Diese Stellen werden als Originalton bezeichnet, d. h. der an einem Aufnahmeort aufgezeichnete Ton bzw. ein wörtliches Zitat. Der O-Ton ist nicht schriftlich vorbereitet, deshalb verfügt er über wenig klare Merkmale der Mündlichkeit. In dieser Hinsicht gibt es zwei Typen von Kennzeichen der Mündlichkeit: Da sind zum einen die syntaktischen Kennzeichen. Ein Beispiel dafür ist die Wortstellung. Zum anderen das lexikalische Kennzeichnen. Partikel, Adverbien, und deiktische Ausdrücke wie Demonstrativpronomina gelten als Beispiele für den zweiten Typ.

In diesem Zusammenhang vertritt Weinrich (2003) die Ansicht, dass die textkonnektive Hauptfunktion der Demonstrativpronomen dies/er/es in der Rekodierungsleistung liegt. Durch die Verwendung von dieser/es wird auf ein Aufmerksamkeits- und Warnsignal hingewiesen. Dieses Signal gibt dem Hörer einen Hinweis auf „einen Knick in der Referenz“ (vgl. Weinrich 2003: 441). In der mündlichen Orientierung ist das Demonstrativpronomen besonders für die Aufmerksamkeit zuständig, wie im Beispiel Nr. (18) Und genau diese Bahnschienen verliefen früher bis an die Laderampe direkt unter den Fenstern. Das häufige Auftreten der Demonstrativpronomen dieser/es liegt darin begründet, dass die Refokussierung stets notwendig ist, um die gemeinschaftliche Orientierung von Audioguide-Sprecher und Hörer sicherzustellen (vgl. Fandrych/Thumair 2011: 88). Die Beispiele (16), (35) und (37) bestätigen diese Aussage: 
مجلة وادي النيل للاراسات والبحوث الإنسانية والاجتماعية والتربوية (مجلة علمية محكمة)

(ISSN : 2536 - 9555)

(16) Nur diese Zahlen und die Umgebung verraten uns, wofür dieser Raum früher einmal genutzt wurde.

(35) Damit vereint diese Tür sowohl den Wunsch der Gemeinde nach Ästhetik als auch Imam „Kanjus“ theologische Anforderungen an eine Moschee sowie seine Überzeugung vorhandenes Geld eher für Bedürftige auszugeben. (37) Die Menschen kommen an diesen Ort, um zu beten, um zu lernen und um gemeinsam Feste zu feiern.

\section{Schlussbetrachtung}

Der Verfasser hofft, dass die vorliegende Studie zum besseren Verständnis der Textsorte „Audioguide“ beitragen konnte, indem eine im ägyptischen Raum sowohl im schulischen Kontext als auch an der Universität vernachlässigte Textsorte am Beispiel des Audioguides „Früher Lagerhalle, heute Moschee“ beleuchtet wurde. Ein wichtiges Ergebnis der Studie ist die Reichhaltigkeit der Aspekte und Strategien, nach denen ein Audioguide-Text ins Arabische übersetzt werden kann.

Eine weitere wichtige Erkenntnis ist im Stellenwert des Einsatzes dieser Textsorte im ägyptischen Bildungswesen zu sehen, da sie den ägyptischen DaF-Lernenden fremd ist.

Die Analyse der durch die Übersetzung auf Basis deskriptiv gewonnener Daten hat gezeigt, in welchem Ausmaß AudioguideTexte von Aspekten wie Adressatenbezug, räumliche Orientierung und Attribute geprägt sind. Vor diesem Hintergrund soll abschließend die Forderung nach der Konzipierung und Integration der Textsorte "Audioguide“ in die Inhalte des Faches „Übersetzung“ stehen. Dies erscheint meines Erachtens als grundlegendes Anliegen im Lehr-/Lernprozess. In diesem Kontext ist $\mathrm{zu}$ fordern, dieser relativ jungen Textsorte mehr Aufmerksamkeit zu widmen. 
Audioguides. Oder „Mit Ohren sehen“"

Zur Übersetzung der Textsorte „Audioguide“ als Texte der Kunstkommunikation am Beispiel des Textes „Früher Lagerhalle, heute Moschee“6

Dr. Mohamed Nasef

مجلة وادي النيل للاراسات والبحوث الإنسانية والاجتماعية والتربوية (مجلة علمية محكمة)

\section{Literaturverzeichnis}

Bašić, Ema (2020): Übersetzung von Audioguides am Beispiel des Kunsthistorischen Museums Wien und Neandertalermuseums in Krapina. University of Zadar.

Dech, Uwe C. (2004): Aufmerksames Sehen. Konzept einer Audioführung $\mathrm{zu}$ ausgewählten Exponaten. Bielefeld: transcript Verlag.

Fandrych, Christian/ Thurmair, Maria (2011): Textsorten im Deutschen. Linguistische Analysen aus sprachdidaktischer Sicht. Tübingen: Stauffenburg.

Fandrych, Christian/ Thurmair, Maria (2015): Kunst besprechen und vermitteln. Zu sprachlichen Strategien von Audioguides. In: Dobstadt, Michael/ Fandrych, Christian/ Riedner, Renate (Hrsg.): Linguistik und Kulturwissenschaft. $\mathrm{Zu}$ ihrem Verhältnis aus der Perspektive des Faches Deutsch als Fremdund Zeitsprache und anderer Disziplinen. Frankfurt am Main, S. 189-211.

Fandrych, Christian/ Thurmair, Maria (2016): Audioguide. In: Heiko Hausendorf/Marcus Müller (Hg.): Handbuch Sprache in der Kunstkommunikation. Berlin/Boston, S. 380-400.

Hausendorf, Heiko (2005): Die Kunst des Sprechens über Kunst. Zur Linguistik einer riskanten Kommunikationspraxis. In: Klotz, Peter/ Lubkoll, Christine (Hrsg.): Beschreibend wahrnehmen - wahrnehmend beschreiben. Sprachliche und ästhetische Aspekte kognitiver Prozesse. Freiburg im Breisgau, S. 99-134

Hausendorf, Heiko (2011): Kunstkommunikation. In: Habscheid, Stephan (Hrsg.): Textsorten, Handlungsmuster, Oberflächen. Linguistische Typologien der Kommunikation. Berlin/New York, S. 509-535.

Hausendorf, Heiko (2013): je länger man hinschaut - Der Betrachter ist im Audioguide. In: Heike Roll/ Constanze Spieß (Hrsg.): Kunst durch Sprache - Sprache durch Kunst. (Osnabrücker Beiträge zur Sprachtheorie 84). Duisburg, 37-55.

Meier, Christoph (2007): Mediacasting an der Universität St. Gallen: Grundlagen und Szenarien. In: Sabine Seufert, Taiga 
(ISSN : 2536 - 9555)

Brahm: "Ne(x)t Generation Learning". Themenreihe 12. Universität St. Gallen.

Popp, Katrin (2013): Das Bild zum Sprechen bringen. Eine Soziologie des Audioguides in Kunstausstellungen. Bielefeld: transcript.

Reiß, Katharina (1971) Möglichkeiten und Grenzen der Übersetzungskritik. Kategorien und Kriterien für eine sachgerechte Beurteilung von Übersetzungen. München: Hueber.

Reiß Katharina (1976): Texttyp und Übersetzungsmethode. Der operative Text. Kronberg: Scriptor.

Schulze, Holger/ Buhl, Hanna (2012): Eine Ausstellung hören? Über Notwendigkeit und Qualität von Audioguides, in: KunzOtt, Hannelore: Mit den Ohren sehen. Audioguides und Hörstationen in Museen und Ausstellungen. München, S. 2731.

Snell-Hornby, Mary (1999) "Audiomediale Texte". Handbuch Translation. Tübingen: Stauffenburg, 273-274.

Spieß, Constanze (2013): ,Texte im Ohr ${ }^{6}$ - Sprachliche Strategien der Kunstvermittlung im Textformat Audioguide für Kinder und Jugendliche. In: Roll, Heike/ Spieß, Constanze (Hrsg.): Kunst durch Sprache - Sprache durch Kunst (OBST 84), S. $57-74$.

Thurmair, Maria (2015): Überlegungen $\mathrm{zu}$ einer textsortenbezogenen Grammatik. In: Drumbl, Hans/Antonie Hornung (Hrsg.) (2015). IDT 2013 Deutsch von innen. Deutsch von außen. Band 1: Hauptvorträge. Bozen - Bolzano, S. 365-382.

Thurmair, Maria (2018): An der Schnittstelle von DaF und Germanistischer Sprachwissenschaft. Bestandsaufnahme und Perspektiven. In: Wöllstein, Angelika / Gallmann, Peter / Habermann, Mechthild / Krifka, Manfred (Hrsg.): Grammatiktheorie und Empirie in der Germanistischen Linguistik, S. 409-432.

Vorwerk, Ursula/ Medien, Linon (2001): Wie kommt ein Museum $\mathrm{zu}$ einem Audioguide? In: Mittelungen und Berichte 23. Berichte zur Tagung im Filmmuseum Berlin. 